\title{
Heterodisulfide reductase from methanogenic archaea: a new catalytic role for an iron-sulfur cluster
}

\author{
Reiner Hedderich ${ }^{1, *}$, Nils Hamann ${ }^{1}$ and Marina \\ Bennati $^{2}$
}

${ }^{1}$ Max-Planck-Institute for Terrestrial Microbiology, Karl-von-Frisch Strasse, D-35043 Marburg, Germany ${ }^{2}$ Institute of Physical and Theoretical Chemistry and Center for Biomolecular Magnetic Resonance, J.W. Goethe University of Frankfurt, D-60439 Frankfurt/Main, Germany

*Corresponding author
e-mail: hedderic@staff.uni-marburg.de

\begin{abstract}
Heterodisulfide reductase (HDR) from methanogenic archaea is an iron-sulfur protein that catalyzes reversible reduction of the heterodisulfide (CoM-S-S-CoB) of the methanogenic thiol-coenzymes, coenzyme M (CoM-SH) and coenzyme $\mathrm{B}(\mathrm{CoB}-\mathrm{SH})$. Via the characterization of a paramagnetic reaction intermediate generated upon oxidation of the enzyme in the presence of coenzyme M, the enzyme was shown to contain a [4Fe-4S] cluster in its active site that catalyzes reduction of the disulfide substrate in two one-electron reduction steps. The formal thiyl radical generated by the initial one-electron reduction of the disulfide is stabilized via reduction and coordination of the resultant thiol to the [4Fe-4S] cluster.
\end{abstract}

Keywords: disulfide reductase; ferredoxin:thioredoxin reductase; heterodisulfide reductase; iron-sulfur proteins; methanogenic archaea.

\section{Introduction}

Iron-sulfur clusters in biological systems participate in a broad range of functions, including sensing and regulation, redox and non-redox catalysis, structural roles and electron transfer (Beinert, 2000). In recent years, a new function for iron-clusters has been identified in a number of enzymes that utilize a [4Fe-4S] and S-adenosylmethionine (AdoMet) to generate catalytically essential radicals (Fontecave et al., 2001). In other enzymes that catalyze radical reactions, such as benzoyl-CoA reductase, iron-sulfur clusters have also been shown to possess essential functions (Boll et al., 2001).

An additional new role for iron-sulfur clusters has emerged in recent years, as two disulfide reductases, heterodisulfide reductase (HDR) and ferredoxin:thioredoxin reductase (FTR), were found to utilize a [4Fe-4S] cluster to mediate the reductive cleavage of a disulfide substrate in two one-electron reduction steps (for a recent review see Walters and Johnson, 2004). Data obtained with both enzymes strongly indicate that the role of the [4Fe-4S] cluster is to mediate electron transfer to the substrate disulfide and to stabilize the formal thiyl radical that results from initial one-electron reduction of the disulfide via reduction and coordination of one thiol to a site on the resultant oxidized $[4 \mathrm{Fe}-4 \mathrm{~S}]^{3+}$ cluster. HDR and FTR represent evolutionarily distinct enzymes. They are not sequence-related and share no sequence similarity with enzymes belonging to the wellcharacterized family of pyridinenucleotide disulfide oxidoreductases. These latter enzymes catalyze the electron transfer between $\operatorname{NAD}(\mathrm{P})(\mathrm{H})$ and a disulfide/thiol (Williams, 1995; Williams et al., 2000). Thioredoxin reductase, glutathione reductase and lipoamide dehydrogenase are prominent members of this enzyme family. These enzymes are homodimeric flavoenzymes, having a redox-active disulfide and a FAD in each monomer. The flow of electrons is from $\mathrm{NAD}(\mathrm{P}) \mathrm{H}$ to the FAD and from reduced $F A D$ to the active-site disulfide, and finally from the active-site dithiol to the disulfide substrate. This cascade only involves two-electron transfer reactions.

In this review we summarize our current knowledge on heterodisulfide reductase with a special focus on characterization of the active-site iron-sulfur cluster. The enzyme is compared with the non-sequence-related ferredoxin:thioredoxin reductase, which also uses an active-site iron-sulfur cluster to mediate the reduction of a disulfide substrate.

\section{Physiological function of heterodisulfide reductase and basic biochemical properties of the enzyme}

Heterodisulfide reductase plays a key role in the energy metabolism of methanogenic archaea. Methane formation in these organisms is coupled to the formation of the heterodisulfide (CoM-S-S-CoB) of coenzyme M (CoM$\mathrm{SH})$ and coenzyme $\mathrm{B}(\mathrm{CoB}-\mathrm{SH})$ in a reaction catalyzed by methyl-coenzyme M reductase (Thauer, 1998). Heterodisulfide functions as the terminal electron acceptor of an energy-conserving electron transport chain (Hedderich et al., 1998; Deppenmeier et al., 1999). The reducing equivalents required for the reduction are provided either by $\mathrm{H}_{2}$ or by reduced coenzyme $\mathrm{F}_{420}\left(\mathrm{~F}_{420} \mathrm{H}_{2}\right)$ in reactions catalyzed by $\mathrm{F}_{420}$-non-reducing hydrogenase or $\mathrm{F}_{420} \mathrm{H}_{2}$ dehydrogenase, respectively. The reduction of CoM-S-S$\mathrm{CoB}$ is catalyzed by heterodisulfide reductase (HDR), forming CoM-SH and CoB-SH (Figure 1) (Hedderich et al., 1998). The enzyme has been purified and characterized from Methanothermobacter marburgensis (formerly Methanobacterium thermoautotrophicum strain 


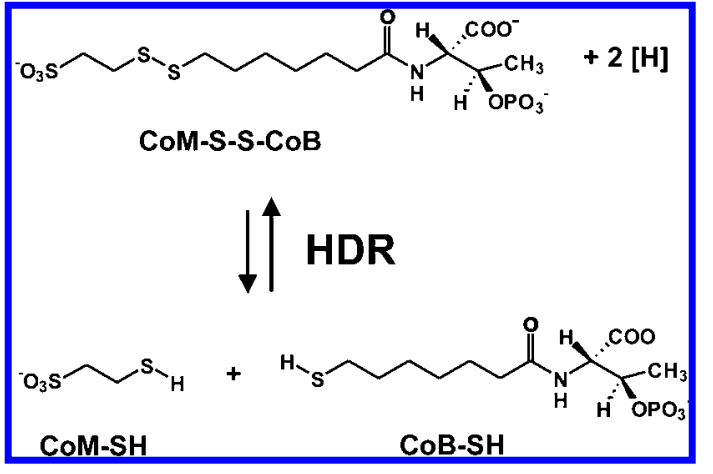

Figure 1 Reaction catalyzed by heterodisulfide reductase and structures of the coenzymes.

Marburg), Methanosarcina barkeri and Methanosarcina thermophila (Hedderich et al., 1990, 1994; Heiden et al., 1994; Künkel et al., 1997; Simianu et al., 1998). Reduction of CoM-S-S-CoB is reversible and HDR exhibits high specificity for its substrates CoM-S-S-CoB or CoM-SH plus CoB-SH.

HDR from $M$. marburgensis is an iron-sulfur flavoprotein that is composed of three different subunits, HdrA, $B$ and $C$ (Figure 2). From the primary sequence it was deduced that HdrA contains a typical FAD-binding motif and four binding motifs for [4Fe-4S] clusters. HdrB contains no characteristic cofactor-binding motif and $\mathrm{HdrC}$ contains two binding motifs for [4Fe-4S] clusters (Hedderich et al., 1994). HDR from the two closely related Methanosarcina species $M$. barkeri and $M$. thermophila

\begin{tabular}{|c|c|c|c|}
\hline \multicolumn{4}{|c|}{ Heterodisulfide reductase $M$. barkeri } \\
\hline HdrE & HdrD & & \\
\hline heme $b$ & $2[4 \mathrm{Fe}-4 \mathrm{~S}]$ & $10 \mathrm{C}$ & \\
\hline \multicolumn{4}{|c|}{ Heterodisulfide reductase $M$. marburgensis } \\
\hline HdrA & HdrC & \multicolumn{2}{|l|}{ HdrB } \\
\hline $4[4 \mathrm{Fe}-4 \mathrm{~S}]$ & $2[4 \mathrm{Fe}-4 \mathrm{~S}][$ & \multicolumn{2}{|l|}{$10 \mathrm{C}$} \\
\hline \multicolumn{4}{|c|}{ Thiol:fumarate reductase $M$. marburgensis } \\
\hline TfrA & \multicolumn{2}{|l|}{ TfrB } & \\
\hline FAD & $\begin{array}{l}\mid 2 \mathrm{Fe}-2 \mathrm{SI} \\
2 \mid 4 \mathrm{Fe}-4 \mathrm{SI}\end{array}$ & $10 \mathrm{C}$ & \\
\hline \multicolumn{4}{|c|}{ Succinate dehydrogenase Sulfolobus species } \\
\hline SdhA & SdhB & SdhC & SdhD \\
\hline FAD & 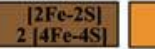 & $10 \mathrm{C}$ & \\
\hline
\end{tabular}

Figure 2 Schematic alignment of heterodisulfide reductase from Methanosarcina barkeri, heterodisulfide reductase from Methanothermobacter marburgensis, and thiol:fumarate reductase from Methanothermobacter marburgensis.

The subunits $\mathrm{HdrD}, \mathrm{HdrCB}$, and $\mathrm{TfrB}$, which show a high degree of sequence similarity, are shown in light brown. In addition to the eight cysteine residues that coordinate the two [4Fe-4S] clusters, these subunits contain 10 conserved cysteine residues $(10 \mathrm{C})$ in two $\mathrm{CX}_{31-32} \mathrm{CCX}_{33-38} \mathrm{CXXC}$ sequence motifs. Subunits HdrE, HdrA, and TfrA have no sequence similarity and have different functions in the different enzymes. For comparison, succinate dehydrogenase from Sulfolobus species is shown. Subunit SdhC of this enzyme is homologous to HdrB. is only composed of two subunits, a membrane-bound b-type cytochrome ( $\mathrm{HdrE})$ and a hydrophilic subunit $(\mathrm{HdrD})$, the latter containing two classical binding motifs for [4Fe-4S] clusters (Figure 2) (Heiden et al., 1994; Künkel et al., 1997; Simianu et al., 1998).

The two types of HDR differ with respect to their physiological electron donor. $M$. barkeri HdrDE receives reducing equivalents from the reduced methanophenazine pool via its $b$-type cytochrome subunit. The enzyme is part of an energy-conserving membrane-bound electron transport chain with $\mathrm{H}_{2}$ or reduced coenzyme $\mathrm{F}_{420}$ as electron donor and the heterodisulfide as terminal electron acceptor (Bäumer et al., 1998; Ide et al., 1999). M. marburgensis HdrABC forms a tight complex with the $\mathrm{F}_{420}$-non-reducing hydrogenase (Mvh) (Setzke et al., 1994; Stojanowic et al., 2003). This six-subunit complex catalyzes the reduction of the heterodisulfide by $\mathrm{H}_{2}$. For none of the subunits of this enzyme complex are transmembrane helices predicted with standard membranetopology prediction programs, and after cell lysis this complex is almost completely localized in the soluble fraction. It is not yet known how this complex is attached to the membrane in vivo and how the exergonic reduction of the heterodisulfide is coupled to energy conservation in M. marburgensis.

For identification of the catalytic subunit of HDR, comparison between both enzymes was quite helpful. Subunit HdrD of the $M$. barkeri enzyme was shown to be a homologue of a hypothetical fusion protein of the M. marburgensis HdrCB subunits (Figure 2). A homologue of the $M$. marburgensis HdrA subunit is lacking in the enzyme from Methanosarcina species, while a homologue of the Methanosarcina HdrE subunit is lacking in the $M$. marburgensis enzyme. It has therefore been concluded that the conserved subunits $\mathrm{HdrD}$ and $\mathrm{HdrCB}$ must harbor the catalytic site for the reduction of the disulfide substrate, while the non-conserved subunits $\mathrm{HdrE}$ and HdrA interact with the physiological electron donor, which differs for the two types of HDR (Künkel et al., 1997). The proposed catalytic subunits $M$. barkeri HdrD and M. marburgensis HdrCB are also sequencerelated to subunit TfrB of thiol:fumarate reductase from Methanothermobacter species, which catalyzes the reduction of fumarate to succinate with $\mathrm{CoM}-\mathrm{SH}$ plus $\mathrm{CoB}-\mathrm{SH}$ as electron donor, thus generating CoM-S-SCoB (Figure 2) (Heim et al., 1998).

HdrB of M. marburgensis HDR contains two copies of a cysteine-rich sequence motif $\left(\mathrm{CX}_{31-32} \mathrm{CCX}_{33-38} \mathrm{CXXC}\right)$. This cysteine motif is also conserved in the C-terminal part of HdrD of $M$. barkeri HDR and in TfrB of thiol: fumarate reductase, as well as in a large number of other enzymes, e.g., succinate dehydrogenase from some archaeal species (Figure 2, Table 2). It has been proposed that part of these cysteine residues coordinate an additional iron-sulfur cluster (Künkel et al., 1997; Heim et al., 1998).

\section{Characterization of HDR by EPR spectroscopy}

The number, type and redox properties of Fe-S clusters in $M$. marburgensis HDR have been investigated by EPR and resonance Raman spectroscopies (Madadi-Kahkesh 
et al., 2001). Oxidized HDR samples exhibited no signals attributable to paramagnetic Fe-S clusters and the resonance Raman spectrum was readily interpreted exclusively in terms of $S=0[4 \mathrm{Fe}-4 \mathrm{~S}]^{2+}$ clusters. In the absence of substrates, dye-mediated EPR redox titrations performed with HDR from $M$. marburgensis revealed a high-potential $[4 \mathrm{Fe}-4 \mathrm{~S}]^{2+,+}$ center $\left(E_{\mathrm{m}}=\right.$ $-153 \mathrm{mV}$ vs. NHE at $\mathrm{pH} 7.6)$ and multiple magnetically interacting low-potential $[4 \mathrm{Fe}-4 \mathrm{~S}]^{2+,+}$ centers $\left(E_{\mathrm{m}}\right.$ $<-300 \mathrm{mV}$ vs. NHE at $\mathrm{pH}$ 7.6). Similar results were obtained for redox titrations on HDR from Methanosarcina thermophila (Simianu et al., 1998), with the two [4Fe$4 \mathrm{~S}]^{2+,+}$ clusters having midpoint potentials of -100 and $-400 \mathrm{mV}$.

EPR signals of potential relevance to the catalytic cycle were observed on reaction of oxidized HDR with either CoM-SH or CoB-SH, the co-substrates for the oxidative reaction (Madadi-Kahkesh et al., 2001). In the presence of CoM-SH, a novel $S=\frac{1}{2}$ resonance was observed at temperatures below $50 \mathrm{~K}$, with principal $g$ values of 2.013, 1.991 and 1.938 (Figure 3). This paramagnetic species has been designated CoM-HDR. The resonance was lost on reduction $\left(E_{\mathrm{m}}=-185 \mathrm{mV}\right.$ vs. $\mathrm{NHE}$ at $\mathrm{pH}$ 7.6) and on reaction with $\mathrm{CoB}-\mathrm{SH}$. Hence, it was attributed to the product of the oxidative half-reaction that occurs in the absence of CoB-SH, in which case it is likely to correspond to a trapped intermediate in the catalytic cycle. A species with similar $g$ values $(g=2.018,1.996$ and 1.954) and relaxation properties was observed when oxidized HDR was reacted with $\mathrm{CoB}-\mathrm{SH}$, designated CoB-HDR. However, redox titrations revealed a significantly higher midpoint potential $\left(E_{\mathrm{m}}=-30 \mathrm{mV}\right.$ vs. NHE at $\mathrm{pH}$ 7.6) for CoB-HDR than the CoM-SH-generated species and argue against a role as an intermediate in the HDR catalytic cycle. HDR from M. barkeri exhibited EPR spectroscopic properties very similar to those of the $M$. marburgensis enzyme after reaction of the oxidized enzyme with $\mathrm{CoM}-\mathrm{SH}$ or $\mathrm{CoB}-\mathrm{SH}$ (Madadi-Kahkesh et al., 2001). The CoM-SH-induced EPR signal in M. marburgensis HDR showed ${ }^{57} \mathrm{Fe}$ broadening, indicating that it corresponds to a paramagnetic iron-sulfur center (Madadi-Kahkesh et al., 2001).

To address the question as to whether $\mathrm{CoM}-\mathrm{SH}$ is attached to this iron-sulfur center in CoM-HDR, CoM${ }^{33} \mathrm{SH}$ was used as the substrate for the enzyme (Duin et al., 2003). With HDR from M. marburgensis and $M$. barkeri, anisotropic broadening of the EPR spectrum was obtained that results from anisotropic hyperfine coupling between the ${ }^{33} \mathrm{~S}$ nucleus, which has a nuclear spin of $3 / 2$, and the paramagnetic center. From EPR simulations, hyperfine coupling constants of 7.0, 5.8, 1.8 G, and 7.0, 5.8 and $3.0 \mathrm{G}$ were estimated for $\mathrm{CoM}-{ }^{33} \mathrm{SH}$-treated $M$. barkeri and M. marburgensis HDR, respectively. The magnitude of this coupling suggests that the sulfur of $\mathrm{CoM}-\mathrm{SH}$ is attached to an iron-site rather than a bridging sulfide.

\section{Selenium K-edge X-ray absorption spectroscopy}

To further investigate the site of CoM-SH binding to the cluster, studies with the substrate analog seleno-coen- zyme M (CoM-Se-) were performed. With HDR from $M$. barkeri and $M$. marburgensis, addition of CoM-Se- to the oxidized enzyme resulted in a new EPR spectrum with principal $g$ values of 2.012, 1.993 and 1.974 (M. marburgensis HDR) and 2.012, 1.993 and 1.973 (M. barkeri HDR) (Figure 3; Duin et al., 2003). These signals could be observed under non-saturating conditions between 5 and $30 \mathrm{~K}$. Kinetic studies confirmed that HDR catalyzes the oxidation of $\mathrm{CoB}-\mathrm{SH}$ and $\mathrm{CoM}-\mathrm{Se}^{-}$to CoM-Se-S$\mathrm{CoB}$, with duroquinone as electron acceptor. The finding that the substrate analog seleno-coenzyme $M$ induces a new EPR signal is again indicative of direct binding of the compound to the active-site cluster of HDR.

To further elucidate the site of cluster attachment, selenium K-edge X-ray absorption spectroscopy (XAS) in combination with the selenium analog of coenzyme $M$ (CoM-Se-) was performed (Shokes et al., 2005). The same method had been used to determine the interaction between Se-adenosyl-L-selenomethionine and lysine 2,3-aminomutase (Cosper et al., 2000).

The best fit of the Se EXAFS data for $\mathrm{HDR}+\mathrm{CoM}-\mathrm{SeH}$ was a model that contains one Se-C bond (at $1.98 \AA$ ) and one Se-Fe interaction at ca. 2.4 $\AA$. A search of the Cambridge Structural Database (Wavefunction Inc.) for Fe-Se-C fragments revealed a chemically reasonable average Se-Fe bond of $2.42 \pm 0.02 \AA$ (Shokes et al., 2005, and references therein), consistent with the determination of $2.41 \AA$ for HDR+CoM-SeH. These data provide a direct structural framework to interpret the EPR experiments on HDR incubated with the substrate CoM- ${ }^{33} \mathrm{SH}$ (Duin et al., 2003). In particular, it provides direct structural evidence of an Fe-Se interaction in HDR reacted with $\mathrm{CoM}-\mathrm{SeH}$.

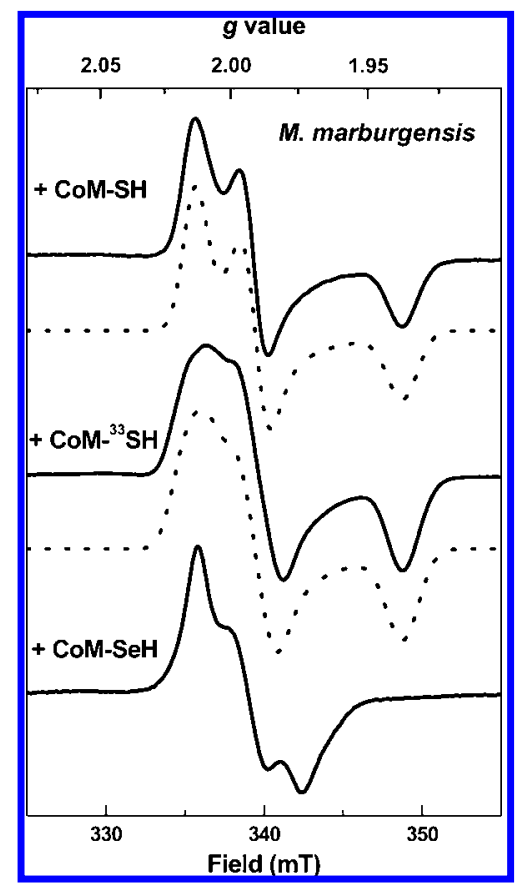

Figure 3 Comparison of EPR spectra of oxidized HDR from $M$. marburgensis.

The enzyme was treated with either CoM-SH containing the natural abundance mixture of sulfur, CoM- ${ }^{33} \mathrm{SH}$ (more than $99 \%$ enriched in ${ }^{33} \mathrm{~S}$ ) or CoM-Se- (from Duin et al., 2003). The dotted lines are EPR simulations. 


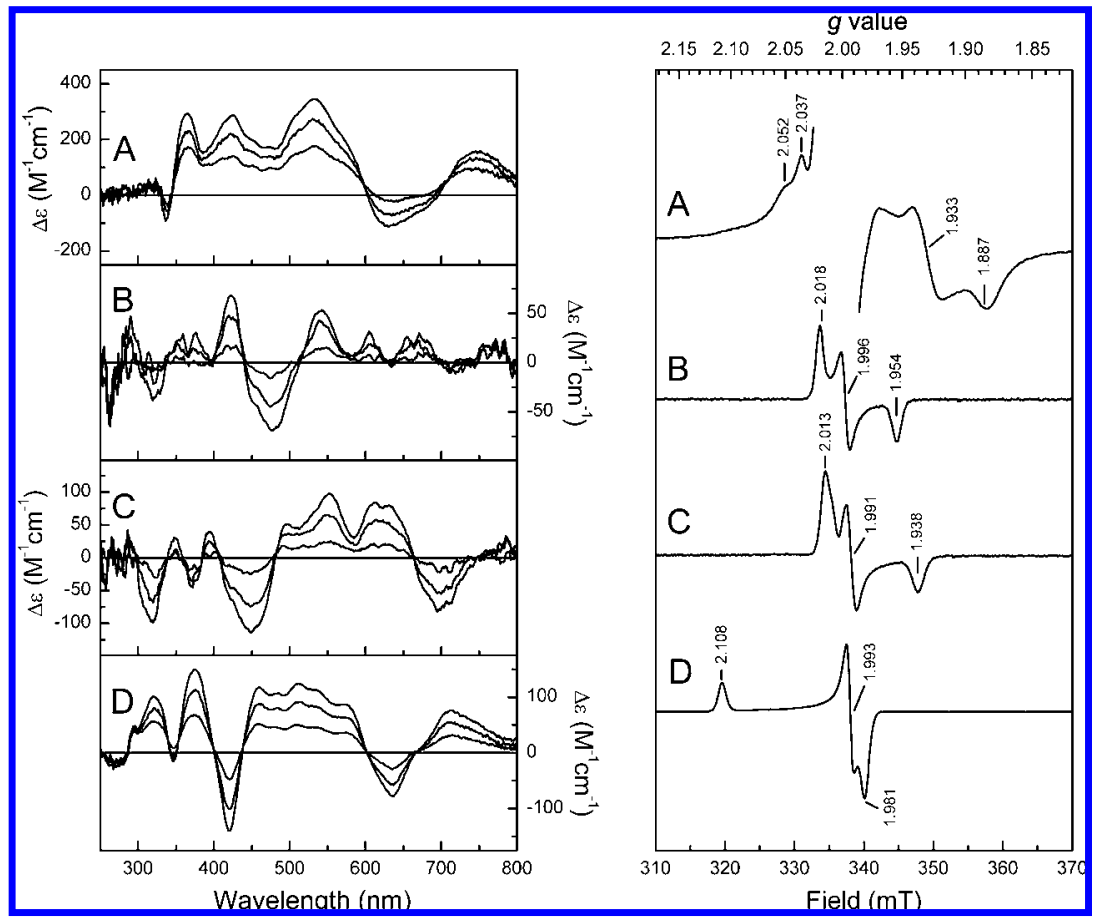

Figure 4 Comparison of the VTMCD and EPR spectra of different forms of HDR from M. marburgensis, and NEM-FTR from Synechocystis sp. PCC6803.

Left panel: VTMCD spectra recorded at 1.7, 4.2 and $10 \mathrm{~K}$ with an applied magnetic field of $6 \mathrm{~T}$. All transitions increase in intensity with decreasing temperature. Right panel: EPR spectra recorded at $5 \mathrm{~K}(\mathrm{~A})$ and $30 \mathrm{~K}(\mathrm{~B}-\mathrm{D})$ with $2 \mathrm{~mW}$ of microwave power and modulation amplitude of $0.6 \mathrm{mT}$ at a microwave frequency of $9.43 \mathrm{GHz}$. (A) Dithionite-reduced HDR; (B) CoB-HDR, duroquinoneoxidized HDR incubated with CoB-SH; (C) CoM-HDR, duroquinone-oxidized HDR incubated with CoM-SH; (D) oxidized NEM-FTR (from Duin et al., 2002).

\section{Characterization of the active-site Fe-S cluster in HDR by VTMCD spectroscopy}

To elucidate the type of $\mathrm{Fe}-\mathrm{S}$ cluster present in the active-site of HDR, the enzyme was also studied by variable-temperature magnetic circular dichroism (VTMCD; Duin et al., 2002). Only paramagnetic metal chromophores exhibit intense VTMCD bands and this allowed characterization of the ground- and excited-state properties of paramagnetic Fe-S centers in HDR samples, in which the absorption spectrum is dominated by contributions from diamagnetic Fe-S clusters and FAD (Johnson et al., 1999). CoM-HDR and CoB-HDR were generated by reacting the oxidized enzyme with either CoM-SH or CoB-SH. The absence of additional EPR signals at lower temperatures indicated that all the $[4 \mathrm{Fe}-4 \mathrm{~S}]^{2+,+}$ clusters were oxidized and therefore diamagnetic (Madadi-Kahkesh et al., 2001). Hence, the VTMCD spectra of CoM-HDR (Figure 4C, left panel) and CoB-HDR (Figure 4B, left panel) are expected to facilitate selective characterization of the electronic transitions associated with the Fe-S centers responsible for these novel EPR signals. This was confirmed by variable-temperature, variable-field MCD saturation magnetization studies, which indicated that each of the temperaturedependent MCD bands of CoM-HDR and CoB-HDR originated exclusively from the $S=\frac{1}{2}$ ground state responsible for the EPR signals. The VTMCD spectra of both CoB-HDR and CoM-HDR were different from each other and both were distinct from those associated with the most common types of oxidized paramagnetic Fe-S clusters, i.e., cubane and linear $[3 \mathrm{Fe}-4 \mathrm{~S}]^{+}$clusters and HiPIP-type [4Fe-4S $]^{3+}$ clusters (Johnson et al., 1999). However, the VTMCD spectra of CoM-HDR and CoBHDR both showed correspondence to that observed for the novel type of $[4 \mathrm{Fe}-4 \mathrm{~S}]^{3+}$ cluster found in $\mathrm{N}$-ethylmaleimide-modified ferredoxin:thioredoxin reductase (NEM-FTR; see below) (Staples et al., 1996, 1998; Figure 4D, left panel). The similarity was particularly striking on comparison of the VTMCD spectra of CoM-HDR and NEM-FTR. Both have the same pattern of positive and negative bands, with equivalent bands shifted to lower energy by approximately $2000 \mathrm{~cm}^{-1}$ in CoM-HDR. The correspondence is less immediately apparent between the VTMCD spectra of CoB-HDR and NEM-FTR due to changes in the relative intensities of the MCD bands and a further red shift of each of the bands. Nevertheless, the pattern of VTMCD bands observed in NEM-FTR is clearly preserved in both CoM-HDR and CoB-HDR, indicating that each contains a similar type of novel $[4 \mathrm{Fe}-4 \mathrm{~S}]^{3+}$ cluster.

\section{${ }^{57} \mathrm{Fe}-E N D O R$ evidence for an unusual [4Fe-4S] cluster in the active-site of HDR}

${ }^{57} \mathrm{Fe}$ pulsed ENDOR spectroscopy was used to identify the iron sites of the active cluster (Bennati et al., 2004) and to gain more insight into the structure of the CoMHDR paramagnetic intermediate. The experiments were performed at two very different frequencies, 9 and $94 \mathrm{GHz}$, and consistently showed two pairs of doublets 


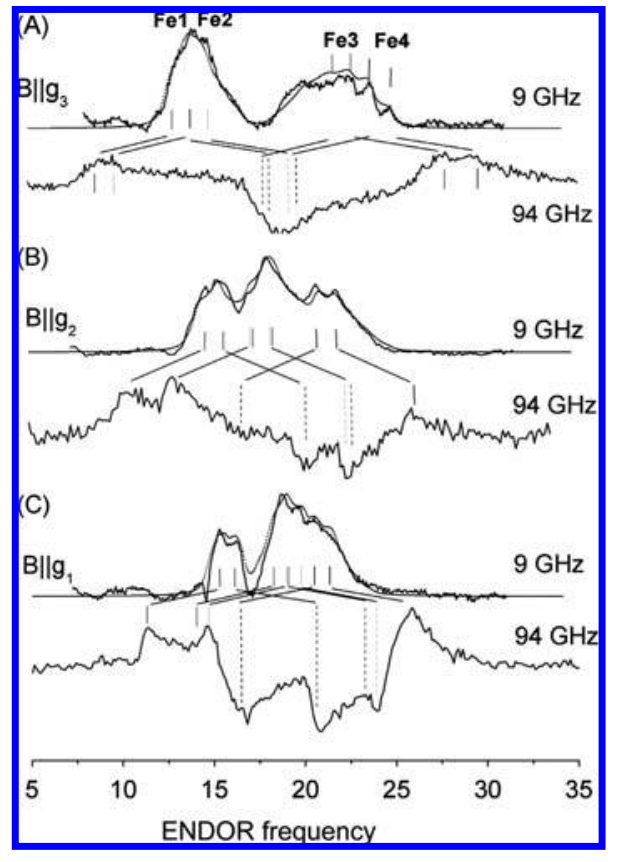

Figure 5 Davies ENDOR spectra of ${ }^{57} \mathrm{Fe}$-enriched CoM-HDR recorded at 9 and $94 \mathrm{GHz}$ at different positions of the EPR line according to $B\left\|g_{3}(A), B\right\| g_{2}(B)$ and $B \| g_{1}(C)$.

Simulations are displayed as dotted lines. The $9-\mathrm{GHz}$ spectra were recorded by strongly attenuating the overlapping ${ }^{1} \mathrm{H}$ resonances with hard microwave preparation pulses. The remaining features of the ${ }^{1} \mathrm{H}$ resonances were subtracted after a reference measurement (from Bennati et al., 2004).

arising from four distinct iron sites. The multi-frequency approach allowed quantitative analysis with spectral simulation and assignment of all resonances observed. The spectra recorded at the three canonical positions of the EPR line (i.e., $B$ parallel to $g_{3}, g_{2}$ and $g_{1}$ ) are displayed in Figure 5, together with the simulations. Furthermore, the ENDOR spectra at $94 \mathrm{GHz}$ displayed absorptive and emissive lines due to thermal electron-spin polarization. The pattern observed allowed the sign of the hyperfine coupling at each iron site to be determined.

The hyperfine tensors obtained from the simulation and from the sign of the hyperfine couplings gave rise to the isotropic hyperfine constants reported in Table 1. For comparison, we also report the hyperfine tensors of a typical four-fold cysteine-coordinated $[4 \mathrm{Fe}-4 \mathrm{~S}]^{3+}$ cluster in a model compound (Rius and Lamotte, 1989), which

Table 1 Isotropic ${ }^{57} \mathrm{Fe}$ hyperfine couplings of CoM-HDR (Bennati et al., 2004) compared to a $[4 \mathrm{Fe}-4 \mathrm{~S}]^{3+}$ cluster in a model system from a single crystal ENDOR study (Rius and Lamotte, 1989), in a HiPIP protein (Kappl, 1999) and to the cluster intermediate in NEM-FTR (Jameson et al., 2003).

\begin{tabular}{lcccc}
\hline Fe site & \multicolumn{4}{c}{$a_{\text {iso }}(\mathrm{MHz})$} \\
\cline { 2 - 5 } & $\begin{array}{c}\text { Model } \\
\text { system }\end{array}$ & $\begin{array}{c}\text { HiPIP } \\
\text { protein }\end{array}$ & NEM-FTR & CoM-HDR \\
\hline 1 & 17.4 & 21.6 & 22 & 29 \\
2 & 19.8 & 21.6 & 27 & 33.3 \\
3 & -32.7 & -33 & -37 & -39.2 \\
4 & -33.5 & -33 & -37 & -43.4 \\
\hline
\end{tabular}

The data for NEM-FTR were extracted from the Mössbauer data. is very similar to those found in HiPIP proteins (Kappl et al., 1999). We also used the hyperfine tensors obtained for NEM-FTR for comparison (Jameson et al., 2003). The ${ }^{57} \mathrm{Fe}$ couplings of the $[4 \mathrm{Fe}-4 \mathrm{~S}]^{3+}$ model compound and of the HiPIP protein are systematically lower than in CoM-HDR. Thus, the results suggest that the couplings in CoM-HDR reflect the interaction of the cluster with the substrate, which is absent in HiPIP proteins or model compounds. Enhanced ${ }^{57} \mathrm{Fe}$ couplings were also observed in NEM-FTR and could be indicative of the similarity between the two cluster species, as proposed from the previous EPR (Madadi-Kahkesh et al., 2001; Duin et al., 2003) and VTMCD (Duin et al., 2002) data. Nevertheless, in NEM-FTR, pronounced anisotropy (ca. $66 \%$ ) of the ${ }^{57} \mathrm{Fe}$ coupling was reported at a unique site (Jameson et al., 2003), which was not observed in CoMHDR (Bennati et al., 2004).

\section{Comparative studies with ferredoxin:thioredoxin reductase (FTR)}

FTR catalyzes the reduction of a disulfide on the protein substrate, thioredoxin, in two one-electron steps, using reduced [2Fe-2S] ferredoxin as the electron donor. The enzyme is found in chloroplasts and cyanobacteria and plays a central role in light regulation of the activity of enzymes involved in oxygenic photosynthesis (Walters and Johnson, 2004).

FTR is a $\alpha \beta$-heterodimer composed of a highly conserved $13-\mathrm{kDa}$ catalytic $\beta$-subunit and a variable $\alpha$-subunit of similar or smaller mass. The catalytic subunit contains a redox-active disulfide and a [4Fe-4S] cluster as the sole prosthetic group (Droux et al., 1987). Extensive spectroscopic studies using EPR, resonance Raman, VTMCD, ENDOR and Mössbauer spectroscopies have been performed with $\mathrm{N}$-ethylmaleimide-modified FTR (NEM-FTR) from spinach, in which one of the cysteines that form the redox-active disulfide is selectively alkylated (Staples et al., 1996, 1998). NEM-FTR was found to be paramagnetic in the oxidized state and exhibited a near-axial EPR signal $(g=2.112,1.997$ and 1.984 for spinach NEM-FTR). The resonance was lost upon dithionite reduction in a reversible one-electron process, with a midpoint potential of $-210 \mathrm{mV}$. Under turnover conditions, non-alkylated FTR showed the same EPR signal as observed for the cluster in NEM-FTR, indicating that the latter species is a stable analog of a one-electronreduced enzymatic intermediate. Spectroscopic characterization of the paramagnetic species observed in NEM-FTR strongly indicates the presence of a $[4 \mathrm{Fe}-4 \mathrm{~S}]^{3+}$ cluster with anomalous EPR and MCD properties and with a 500-600-mV decrease in redox potential compared to the $[4 \mathrm{Fe}-4 \mathrm{~S}]^{3+}$ cluster in oxidized HiPIPs. Furthermore, Mössbauer experiments (Jameson et al., 2003) revealed ${ }^{57} \mathrm{Fe}$ hyperfine couplings, reported here in Table 1 , which indicated unusually large anisotropy at only one Fe site. These anomalous properties have been interpreted in terms of a $[4 \mathrm{Fe}-4 \mathrm{~S}]^{3+}$ cluster with a unique fivecoordinate $\mathrm{Fe}$ site with two cysteinate ligands. In the catalytic cycle, one-electron reduction of the redoxactive disulfide generates a thiol and a thiyl radical, with the latter stabilized by reduction and coordination to the 
$[4 \mathrm{Fe}-4 \mathrm{~S}]$ cluster and generating the unique $[4 \mathrm{Fe}-4 \mathrm{~S}]^{3+}$ center.

The crystal structure is known for FTR from Synechocystis sp. PCC6803. The structure shows a cysteine disulfide asymmetrically positioned relative to the cluster, with one of the $S$ atoms at a distance of $3.1 \AA$ from both a cluster $\mathrm{Fe}$ and the $\mathrm{S}$ atom of a cluster-ligating cysteinate, and $3.5 \AA$ from a cluster $\mu_{3}-S$ atom. The crystal structure suggests that a cluster $\mathrm{Fe}$ is the most likely point of attachment to yield an intermediate involving a [4Fe-4S] cluster with a five-coordinate Fe site (Dai et al., 2000).

For HDR, several lines of evidence argue in favor of a mechanism involving direct interaction of the heterodisulfide substrate with the active-site [4Fe-4S] cluster rather than the FTR-type mechanism, in which cleavage of the substrate disulfide by the [4Fe-4S] cluster is mediated by an active-site disulfide in close proximity to the cluster. First, the experiments with $\mathrm{CoM}-{ }^{3} \mathrm{SH}$ provide strong evidence for direct binding of the substrate to the cluster. Second, the $[4 \mathrm{Fe}-4 \mathrm{~S}]^{3+}$ species in HDR are readily formed under oxidizing conditions on addition of exogenous thiols such as CoM-SH, CoB-SH, DTT or $\beta$-mercaptoethanol (Madadi-Kahkesh et al., 2001). This does not occur in FTR, since the active-site disulfide that is present in oxidized samples can only be cleaved under reducing conditions using the physiological electron donor, reduced ferredoxin, or mediator dyes such as reduced viologens (Schürmann et al., 1995). The [4Fe$4 S]^{3+}$ species in FTR is only observed as a stable species on oxidation when one of the active-site cysteine residues has been alkylated, and therefore is not available to reform the active-site disulfide on oxidation, leaving the free cysteine available to interact with the cluster. Third, M. marburgensis Hdr is not inhibited by cysteine alkylating reagents at concentrations up to $2 \mathrm{~mm}$ (Madadi-Kahkesh et al., 2001), whereas cysteine alkylating reagents are potent inhibitors of FTR as a result of alkylation of the interchange thiol of the active-site disulfide (Staples et al., 1996).

\section{Subunit HdrB of M. marburgensis HDR contains the active-site iron-sulfur cluster}

To elucidate if the unique cysteine motif present in HdrB and in the C-terminal part of $\mathrm{HdrD}$ (Figure 2) binds the active-site iron-sulfur cluster, HdrB was produced in a heterologous host. HdrB was chosen since it does not contain a classic [4Fe-4S] cluster-binding motif (CxxCxxCxxxCP), which could interfere with the spectroscopic characterization. Initial expression experiments of $h d r B$ in $B$. subtilis and $E$. coli resulted in a brown protein with a content of $2 \mathrm{~mol}$ non-heme iron and $2 \mathrm{~mol}$ of acidlabile sulfur per mol protein (Madadi-Kahkesh et al., 2001). UV/visible and resonance Raman (RR) spectroscopies showed that HdrB thus produced contains a [2Fe-2S] cluster. This cluster was, however, labile and was destroyed upon reduction by sodium dithionite, as indicated by the loss of absorbance in the visible region. The RR spectrum of as-isolated HdrB showed an absorption band at $290 \mathrm{~cm}^{-1}$ characteristic for [2Fe-2S] clusters. In native HDR, this absorption band at 290 $\mathrm{cm}^{-1}$ was not observed. It was therefore concluded that the [2Fe-2S] cluster found in $\mathrm{HdrB}$ is an artifact of the heterologous expression system. When, however, the heterologously produced protein was incubated in vitro under anaerobic conditions in the presence of $\mathrm{Fe}^{2+}, \mathrm{Na}_{2} \mathrm{~S}$ and cysteine, the UV/visible absorption spectrum with maxima at 320, 420 and $460 \mathrm{~nm}$ changed to a spectrum with a broad absorbance between 350 and $500 \mathrm{~nm}$. Furthermore, reduction of the cluster by sodium dithionite was now reversible and did not result in cluster breakdown. This protein, when oxidized, exhibited an EPR signal with $g=2.016,1.995$ and 1.952 reminiscent of the CoB-HDR signal (Hamann and Hedderich, unpublished results). In contrast to the native enzyme (HdrABC), which was EPR-silent when oxidized in the absence of its thiol substrates, HdrB showed the characteristic EPR signal upon oxidation of the protein even in the absence of either CoB-SH or CoM-SH. This result is puzzling, since it indicates that $\mathrm{HdrB}$ is able to generate a cluster with similar electronic and structural properties in the absence of substrate. However, native HDR also exhibited a CoM-HDR-like EPR signal when oxidized in the presence of non-substrate thiols such as mercaptoethanol or cysteine. These paramagnetic species, as CoB$\mathrm{HDR}$, had redox potentials at least $170 \mathrm{mV}$ more positive than CoM-HDR. They may be formed in an artificial side reaction occurring at high redox potential. Since no extra thiol was added during sample preparation of $\mathrm{HdrB}$, it has to be considered that the protein uses an intrinsic cysteine residue as an extra thiol ligand to form the paramagnetic center upon oxidation. HdrB did not catalyze the reduction of CoM-S-S-CoB with reduced benzylviologen as electron donor, nor the oxidation of $\mathrm{CoM}-\mathrm{SH}$ plus $\mathrm{CoB}-\mathrm{SH}$ with methylene blue as electron acceptor. A possible reason could be that $\mathrm{HdrC}$ is required for electron transfer to these redox dyes. Although the paramagnetic species present in HdrB needs to be further characterized (e.g., by ENDOR spectroscopy), the data clearly show that subunit $\mathrm{HdrB}$ binds the active-site ironsulfur cluster, most probably involving the unique Cysmotif (Hamann and Hedderich, unpublished results).

\section{Proposed catalytic mechanism for HDR}

Results obtained from the spectroscopic characterization of CoM-HDR led to the proposal of a mechanistic scheme for the reversible heterodisulfide/dithiol cleavage reaction that is catalyzed by HDR in two one-electron steps (Figure 6; Duin et al., 2002). The reaction cycle is described here for the oxidative reaction. This mechanism involves an EPR-silent $[4 \mathrm{Fe}-4 \mathrm{~S}]^{2+}$ in the resting enzyme (1). Binding of CoM-SH (2) to the active site in the presence of an electron acceptor generates the paramagnetic reaction intermediate CoM-HDR (3). Spectroscopic data clearly indicate that CoM-HDR is a $[4 \mathrm{Fe}-4 \mathrm{~S}]^{3+}$ cluster, with CoM-S- attached to the cluster. EPR studies with $\mathrm{CoM}-{ }^{33} \mathrm{SH}$ and Se-X-ray absorption spectroscopic studies with the substrate analog CoM- 


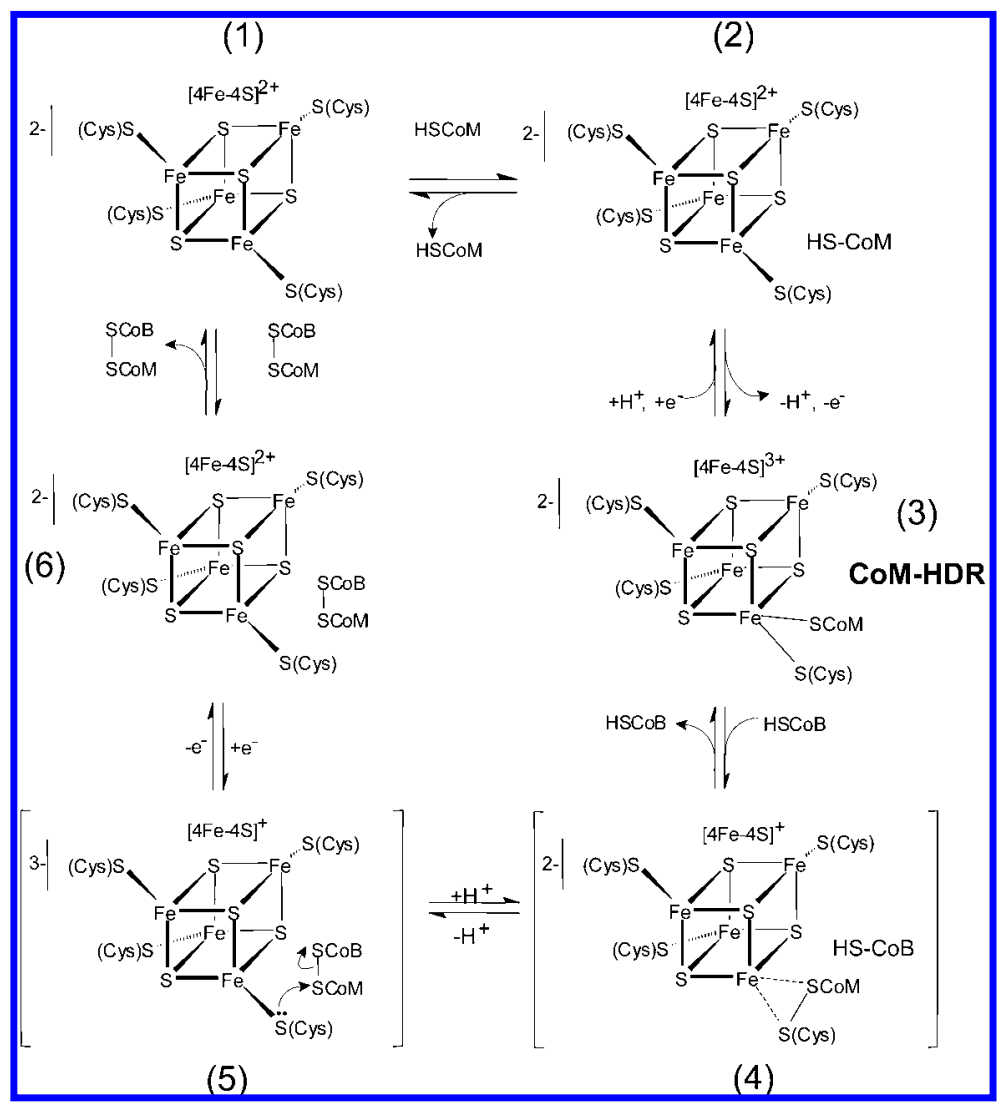

Figure 6 Proposed active-site mechanism for the reversible heterodisulfide/dithiol cleavage reaction catalyzed by HDR.

The oxidation state of the [4Fe-4S] cores is indicated above each of the cubane clusters and the charge of the cluster including the thiolate ligands is indicated to the left of the lines next to the clusters. The different reaction intermediates are numbered. Transient intermediates are shown in brackets and the electron arrows on one of the transient intermediates correspond to the reductase reaction (from Duin et al., 2002). The stable paramagnetic reaction intermediate is labeled as CoM-HDR.

SeH showed that CoM-S- binds to an iron site of the cluster rather than to a cluster $\mu_{3}-\mathrm{S}$. In formal terms, the reaction involves the oxidation of CoM-S- to a thiyl radical, which is stabilized by coordination to the $[4 \mathrm{Fe}-4 \mathrm{~S}]^{2+}$ cluster, resulting in the formation of a $[4 \mathrm{Fe}-4 \mathrm{~S}]^{3+}$ cluster with a CoM-S- ligand. At this stage, it is unclear whether the CoM-S- ligand binds at a unique iron site, replacing one cysteine ligand or generating a five-fold coordinating iron [as shown in Figure 6 (3)]. ${ }^{57} \mathrm{Fe}$ couplings from ENDOR data compared to NEM-FTR and the observation of a cluster species similar to CoM-HDR in isolated HdrB, in the absence of substrate, would be more consistent with the former hypothesis. Nevertheless, more experiments are required to clarify this point. When $\mathrm{CoB}-\mathrm{SH}$ is added to the CoM-HDR reaction intermediate (4 and $\mathbf{5}$ ) in the presence of an electron acceptor, this results in the formation of the diamagnetic $[4 \mathrm{Fe}-4 \mathrm{~S}]^{2+}$ cluster and CoM-S-S-CoB (6). In this series of events, CoB-SH attacks the CoM-thiol group in CoM-HDR in a nucleophilic substitution reaction, forming the disulfide and the cluster in the $1+$ reduced state (5). It is not known if the active-site $[4 \mathrm{Fe}-4 \mathrm{~S}]^{2+}$ cluster is reducible to a stable $[4 \mathrm{Fe}-4 \mathrm{~S}]^{+}$state or if it is immediately reoxidized by the [4Fe-4S] centers in HdrC. The evidence that CoM-Srather than CoB-S- binds to the cluster is based on the observation that the CoM-HDR EPR signal is lost on addition of $\mathrm{CoB}-\mathrm{SH}$ and that the redox potential for CoM-
$\operatorname{HDR}\left(E^{\circ \prime}=-185 \pm 10 \mathrm{mV}\right.$ vs. SHE) is close to the heterodisulfide/dithiol couple ( $E^{\circ \prime}=-143 \pm 10 \mathrm{mV}$ vs. SHE; Tietze et al., 2003).

\section{The catalytic subunit of HDR contains a unique Cys-motif conserved in a large number of proteins}

HdrB of $M$. marburgensis HDR contains two copies of the unique $\mathrm{CX}_{31-32} \mathrm{CCX}_{33-38} \mathrm{CXXC}$ sequence motif, which are also conserved in HdrD of $M$. barkeri HDR and in subunit TfrB of thiol:fumarate reductase (Figure 2). A database search revealed a large number of proteins from non-methanogenic microorganisms with high sequence similarity to $\mathrm{HdrB}, \mathrm{HdrD}$ and TfrB (Table 2). In these proteins at least one of the two $\mathrm{CX}_{31-32} \mathrm{CCX}_{33-38}$ CXXC sequence motifs is conserved. For many of these HDR-related enzymes the catalytic function is not known and they were only identified through genome sequencing projects. Some of these proteins are more closely related to HdrB and lack additional cofactor binding motifs; others are more closely related to HdrD and contain, as HdrD, two four-Cys motifs characteristic for the binding of two [4Fe-4S] clusters in the amino-terminal part of the protein. More detailed biochemical studies have been performed with HDR-related enzymes from 
Table 2 Gene products with significant sequence similarity to the postulated catalytic subunit HdrD of the M. barkeri heterodisulfide reductase, the postulated catalytic subunits $\mathrm{HdrCB}$ of the $M$. thermoautotrophicum heterodisulfide reductase and the subunit TfrB of thiol:fumarate reductase.

\begin{tabular}{|c|c|c|c|c|}
\hline Gene & Organism & Comment/putative transcriptional unit & Sequence identity to & Reference \\
\hline AF 0506 & Archaeoglobus fulgidus & - & HdrD (34\% from 385 aa) & Klenk et al., 1997 \\
\hline AF 0547 & Archaeoglobus fulgidus & $\begin{array}{l}\text { Putative operon with a gene encoding } \\
\text { a } b \text {-type cytochrome }\end{array}$ & $\operatorname{HdrD}$ (23\% from 431 aa) & Klenk et al., 1997 \\
\hline AF 0502 & Archaeoglobus fulgidus & $\begin{array}{l}\text { Hme complex, operon with genes } \\
\text { encoding Fe/S proteins and } b \text {-type } \\
\text { cytochromes }\end{array}$ & $\operatorname{HdrD}(32 \%$ from 149 aa) & Mander et al., 2002 \\
\hline AF 0543 & Archaeoglobus fulgidus & Most similar to $h m c 6$ from $D$. vulgaris & HdrD (20\% from 354 aa) & Klenk et al., 1997 \\
\hline AF 0544 & Archaeoglobus fulgidus & Most similar to $h m c 6$ from $D$. vulgaris & HdrD (20\% from 338 aa) & Klenk et al., 1997 \\
\hline AF 1375 & Archaeoglobus fulgidus & Mvh:Hdl complex & HdrB (30\% from 300 aa) & Mander et al., 2004 \\
\hline$y w j F$ & Bacillus subtilis & $\begin{array}{l}\text { Putative operon with acd } A \text { encoding } \\
\text { acyl-CoA dehydrogenase }\end{array}$ & HdrD (28\% from 390 aa) & Kunst et al., 1997 \\
\hline$h d r D$ & Aquifex aeolicus & Hydrogenase operon & HdrD (25\% from 409 aa) & Deckert et al., 1998 \\
\hline$h d r B$ & Aquifex aeolicus & hdrABC operon & $\mathrm{HdrB}(30 \%$ from $300 \mathrm{aa})$ & Deckert et al., 1998 \\
\hline isp2 & Thiocapsa roseopersicina & Hydrogenase operon & HdrD (23\% from 398 aa) & Rakhely et al., 1998 \\
\hline$s d h C$ & Sulfolobus acidocaldarius & Succinate dehydrogenase operon & HdrB (31\% from 277 aa) & Janssen et al., 1997 \\
\hline$h d r B$ & Synechocystis sp. & - & HdrB (33\% from 300 aa) & Nakamura et al., 1998 \\
\hline hmc6 & Desulfovibrio vulgaris & $\begin{array}{l}\text { hmc operon; encodes a multisubunit } \\
\text { membrane complex }\end{array}$ & HdrD (33\% from 103 aa) & Rossi et al., 1993 \\
\hline$d s r k$ & Allochromatium vinosum & dsr locus encoding sulfite reductase & $\mathrm{HdrD}$ (23\% from $437 \mathrm{aa})$ & Pott and Dahl, 1998 \\
\hline$g / p C$ & Escherichia coli & $\begin{array}{l}\text { Subunit of anaerobic glycerol-3- } \\
\text { phosphate dehydrogenase }\end{array}$ & $\operatorname{HdrD}(27 \%$ from 251 aa) & Cole et al., 1988 \\
\hline$g / c F$ & Escherichia coli & Subunit of glycolate oxidase & & Pellicer et al., 1996 \\
\hline
\end{tabular}

Note that most of the genes are derived from genome sequencing projects and that the function of these genes and their gene products are unknown. The table only gives a selection of proteins or gene products. The conserved domain present in these proteins has been designated as domain of unknown function (DUF) 224 in the Interpro database.

Archaeoglobus species, which are sulfate-reducing archaea. Membrane-bound enzymes have been isolated from $A$. fulgidus and $A$. profundus, designated $\mathrm{Hme}$ (HDR-like menaquinol-oxidizing enzyme), that contain a HdrD-related subunit (Mander et al., 2002, 2004). Hme is membrane-bound and functions as menaquinol:acceptor oxidoreductase. Hme, when oxidized, exhibits an EPR signal with similar $g$ values (e.g., $g=2.031,1.995$, and 1.951 for $A$. fulgidus $\mathrm{Hme}$ ) and relaxation properties to CoM-HDR or CoB-HDR. This paramagnetic species could be reduced in a one-electron reduction step to a diamagnetic form with a midpoint potential of $+100 \mathrm{mV}$. A second enzyme complex was isolated from $A$. profundus, designated Mvh:Hdl complex, containing a HdrBrelated subunit (Mander et al., 2004). In the oxidized state the enzyme complex exhibited a rhombic EPR signal with $g_{\mathrm{xyz}}=2.014,1.939$ and 1.895. In these HDR-like enzymes the characteristic CoM-HDR-like EPR spectrum is generated upon oxidation of the enzymes, even in the absence of substrate. This is a major difference to HDR. The physiological substrate of these enzymes is not known. It has been proposed that they reduce an as yet unknown disulfide substrate, which could then donate reducing equivalents to the enzymes of sulfate reduction. CoM-SH and CoB-SH are not present in Archeoglobus species. Hme-related enzymes are also present in sulfate-reducing bacteria, e.g., Desulfovibrio species (Rossi et al., 1993).

More detailed biochemical studies have also been performed with some unusual archaeal succinate dehydrogenases, e.g., the enzymes from Sulfolobus acidocaldarius and Acidianus ambivalens (Janssen et al., 1997;
Lemos et al., 2001). These enzymes contain a subunit (SdhC) that is sequence-related to HdrB. They lack, however, the characteristic heme $b$-containing membraneanchor protein found in other succinate dehydrogenases and fumarate reductases. It has therefore been proposed that in these enzymes SdhC functions as a membrane anchor that reduces the quinone of these organisms (Lemos et al., 2001). Production of SdhC from Sulfolobus tokodaii strain 7 in $E$. coli resulted in a protein containing a [2Fe-2S] cluster, which was, however, destroyed upon reduction (Iwasaki et al., 2002). The same properties were found for $\mathrm{HdrB}$ when produced in $E$. coli as outlined above. In the case of HdrB, a protein that most likely contains a [4Fe-4S] cluster was only obtained after in vitro reconstitution. This might also be true for SdhC.

A common feature of these HdrB- and HdrD-related proteins could be their ability to ligate a [4Fe-4S] cluster using the unique Cys-motif. In some enzymes this cluster could function as a one-electron/two-electron switch similar to flavins, and could thus catalyze the reduction of a disulfide in two one-electron reduction steps. In other enzymes, such as the archaeal succinate dehydrogenase, it is very unlikely that the enzyme reduces a disulfide. Here, reduction of a quinone is the most likely function. Given the high similarity between these succinate dehydrogenases and thiol:fumarate reductase from methanogens, it is also conceivable that archaeal succinate dehydrogenases are evolutionarily derived from thiol:fumarate reductase. Hence, the carboxyterminal domain of subunit TfrB of thiol:fumarate reductase (Figure 2) may have been converted into a quinone-reducing subunit when aerobic respiration became available. 


\section{Acknowledgments}

This work was supported by the Deutsche Forschungsgemeinschaft Schwerpunktprogramm 1071.

\section{References}

Bäumer, S., Murakami, E., Brodersen, J., Gottschalk, G., Ragsdale, S.W., and Deppenmeier, U. (1998). The $\mathrm{F}_{420} \mathrm{H}_{2}$ : heterodisulfide oxidoreductase system from Methanosarcina species. 2-Hydroxyphenazine mediates electron transfer from $\mathrm{F}_{420} \mathrm{H}_{2}$ dehydrogenase to heterodisulfide reductase. FEBS Lett. 428, 295-298.

Beinert, H. (2000). Iron-sulfur proteins: ancient structures, still full of surprises. J. Biol. Inorg. Chem. 5, 2-15.

Bennati, M., Weiden, N., Dinse, K.P., and Hedderich, R. (2004). ${ }^{57} \mathrm{Fe}$ ENDOR spectroscopy on the iron-sulfur cluster involved in substrate reduction of heterodisulfide reductase. J. Am. Chem. Soc. 126, 8378-8379.

Boll, M., Fuchs, G., and Lowe, D.J. (2001). Single turnover EPR studies of benzoyl-CoA reductase. Biochemistry 40, 7612-7620.

Cole, S.T., Eiglmeier, K., Ahmed, S., Honore, N., Elmes, L., Anderson, W.F., and Weiner, J.H. (1988). Nucleotide sequence and gene-polypeptide relationships of the glpABC operon encoding the anaerobic sn-glycerol-3-phosphate dehydrogenase of Escherichia coli K-12. J. Bacteriol. 170, 2448-2456.

Cosper, N.J., Booker, S.J., Ruzicka, F., Frey, P.A., and Scott, R.A. (2000). Direct $\mathrm{FeS}$ cluster involvement in generation of a radical in lysine 2,3-aminomutase. Biochemistry 39, 15668-15673.

Dai, S., Schwendtmayer, C., Schurmann, P., Ramaswamy, S., and Eklund, H. (2000). Redox signaling in chloroplasts: cleavage of disulfides by an iron-sulfur cluster. Science 287, 655-658.

Deckert, G., Warren, P.V., Gaasterland, T., Young, W.G., Lenox, A.L., Graham, D.E., Overbeek, R., Snead, M.A., Keller, M., Aujay, M., et al. (1998). The complete genome of the hyperthermophilic bacterium Aquifex aeolicus. Nature 392, 353-358.

Deppenmeier, U., Lienard, T., and Gottschalk, G. (1999). Novel reactions involved in energy conservation by methanogenic archaea. FEBS Lett. 457, 291-297.

Droux, M., Jacquot, J.P., Miginac-Maslow, M., Gadal, P., Huet, J.C., Crawford, N.A., Yee, B.C., and Buchanan, B.B. (1987). Ferredoxin-thioredoxin reductase, an iron-sulfur enzyme linking light to enzyme regulation in oxygenic photosynthesis: purification and properties of the enzyme from C3, C4, and cyanobacterial species. Arch. Biochem. Biophys. 252, 426-439.

Duin, E.C., Madadi-Kahkesh, S., Hedderich, R., Clay, M.D., and Johnson, M.K. (2002). Heterodisulfide reductase from Methanothermobacter marburgensis contains an active-site [4Fe$4 \mathrm{~S}$ ] cluster that is directly involved in mediating heterodisulfide reduction. FEBS Lett. 512, 263-268.

Duin, E.C., Bauer, C., Jaun, B., and Hedderich, R. (2003). Coenzyme $\mathrm{M}$ binds to a $[4 \mathrm{Fe}-4 \mathrm{~S}]$ cluster in the active site of heterodisulfide reductase as deduced from EPR studies with the $\left.{ }^{33} \mathrm{~S}\right]$ coenzyme M-treated enzyme. FEBS Lett. 538, 81-84.

Fontecave, M., Mulliez, E., and Ollagnier-de-Choudens, S. (2001). Adenosylmethionine as a source of $5^{\prime}$-deoxyadenosyl radicals. Curr. Opin. Chem. Biol. 5, 506-511.

Hedderich, R., Berkessel, A., and Thauer, R.K. (1990). Purification and properties of heterodisulfide reductase from Methanobacterium thermoautotrophicum (strain Marburg). Eur. J. Biochem. 193, 255-261.
Hedderich, R., Koch, J., Linder, D., and Thauer, R.K. (1994). The heterodisulfide reductase from Methanobacterium thermoautotrophicum contains sequence motifs characteristic of pyridine nucleotide-dependent thioredoxin reductases. Eur. J. Biochem. 225, 253-261.

Hedderich, R., Klimmek, O., Kröger, A., Dirmeier, R., Keller, M., and Stetter, K.O. (1998). Anaerobic respiration with elemental sulfur and with disulfides. FEMS Microbiol. Rev. 22, 353-381.

Heiden, S., Hedderich, R., Setzke, E., and Thauer, R.K. (1994). Purification of a two-subunit cytochrome- $b$-containing heterodisulfide reductase from methanol-grown Methanosarcina barkeri. Eur. J. Biochem. 221, 855-861.

Heim, S., Künkel, A., Thauer, R.K., and Hedderich, R. (1998). Thiol:fumarate reductase (Tfr) from Methanobacterium thermoautotrophicum. Identification of the catalytic sites for fumarate reduction and thiol oxidation. Eur. J. Biochem. 253, 292-299.

Ide, T., Bäumer, S., and Deppenmeier, U. (1999). Energy conservation by the $\mathrm{H}_{2}$ :heterodisulfide oxidoreductase from Methanosarcina mazei Gö1: identification of two protontranslocating segments. J. Bacteriol. 181, 4076-4080.

Iwasaki, T., Kounosu, A., Aoshima, M., Ohmori, D., Imai, T., Urushiyama, A., Cosper, N.J., and Scott, R.A. (2002). Novel [2Fe-2S]-type redox center C in SdhC of archaeal respiratory complex II from Sulfolobus tokodaii strain 7. J. Biol. Chem. 277, 39642-39648.

Jameson, G.N., Walters, E.M., Manieri, W., Schurmann, P., Johnson, M.K., and Huynh, B.H. (2003). Spectroscopic evidence for site specific chemistry at a unique iron site of the [4Fe4S] cluster in ferredoxin:thioredoxin reductase. J. Am. Chem. Soc. $125,1146-1147$.

Janssen, S., Schafer, G., Anemüller, S., and Moll, R. (1997). A succinate dehydrogenase with novel structure and properties from the hyperthermophilic archaeon Sulfolobus acidocaldarius: genetic and biophysical characterization. J. Bacteriol. 179, 5560-5569.

Johnson, M.K., Duderstadt, R.E., and Duin, E.C. (1999). Biological and synthetic $\left[\mathrm{Fe}_{3} \mathrm{~S}_{4}\right]$ clusters. In: Advances in Inorganic Chemistry, A.G. Sykes and R. Cammack, eds. (New York, USA: Academic Press), pp. 1-73.

Kappl, R., Ciurli, S., Luchinat, C., and Hüttermann, J. (1999). Probing structural and electronic properties of the oxidized $[4 \mathrm{Fe}-4 \mathrm{~S}]^{3+}$ cluster of Ectothiorhodospira halophila iso-II high-potential iron-sulfur protein by ENDOR spectroscopy. J. Am. Chem. Soc. 121, 1925-1935.

Klenk, H.-P., Clayton, R.A., Tomb, J.F., White, O., Nelson K.E., Ketchum, K.A., Dodson, R.J., Gwinn, M., Hickey, E.K., Peterson, J.D., et al. (1997). The complete genome sequence of the hyperthermophilic, sulphate-reducing archaeon Archaeoglobus fulgidus. Nature 390, 364-370.

Künkel, A., Vaupel, M., Heim, S., Thauer, R.K., and Hedderich, R. (1997). Heterodisulfide reductase from methanol-grown cells of Methanosarcina barkeri is not a flavoenzyme. Eur. J. Biochem. 244, 226-234.

Kunst, F., Ogasawara, N., Moszer, I., Albertini, A.M., Alloni, G. Azevedo, V., Bertero, M.G., Bessieres, P., Bolotin, A., Borchert, S., et al. (1997). The complete genome sequence of the Gram-positive bacterium Bacillus subtilis. Nature 390 , 249-256.

Lemos, R.S., Gomes, C.M., and Teixeira, M. (2001). Acidianus ambivalens complex II typifies a novel family of succinate dehydrogenases. Biochem. Biophys. Res. Commun. 281, 141-150.

Madadi-Kahkesh, S., Duin, E.C., Heim, S., Albracht, S.P.J., Johnson, M.K., and Hedderich, R. (2001). A paramagnetic species with unique EPR characteristics in the active site of heterodisulfide reductase from methanogenic archaea. Eur. J. Biochem. 268, 2566-2577.

Mander, G.J., Duin, E.C., Linder, D., Stetter, K.O., and Hedderich, R. (2002). Purification and characterization of a membrane-bound enzyme complex from the sulfate-reduc- 
ing archaeon Archaeoglobus fulgidus related to heterodisulfide reductase from methanogenic archaea. Eur. J. Biochem. 269, 1895-1904.

Mander, G.J., Pierik, A.J., Huber, H., and Hedderich, R. (2004). Two distinct heterodisulfide reductase-like enzymes in the sulfate-reducing archaeon Archaeoglobus profundus. Eur. J. Biochem. 271, 1106-1116.

Nakamura, Y., Kaneko, T., Hirosawa, M., Miyajima, N., and Tabata, S. (1998). CyanoBase, a www database containing the complete nucleotide sequence of the genome of Synechocystis sp. strain PCC6803. Nucleic Acids Res. 26, 63-67.

Pellicer, M.T., Badia, J., Aguilar, J., and Baldoma, L. (1996). glc locus of Escherichia coli: characterization of genes encoding the subunits of glycolate oxidase and the glc regulator protein. J. Bacteriol. 178, 2051-2059.

Pott, A.S. and Dahl, C. (1998). Sirohaem sulfite reductase and other proteins encoded by genes at the dsr locus of Chromatium vinosum are involved in the oxidation of intracellular sulfur. Microbiology 144, 1881-1894.

Rakhely, G., Colbeau, A., Garin, J., Vignais, P.M., and Kovacs, K.L. (1998). Unusual organization of the genes coding for HydSL, the stable [NiFe]hydrogenase in the photosynthetic bacterium Thiocapsa roseopersicina BBS. J. Bacteriol. 180, 1460-1465.

Rius, G. and Lamotte, B. (1989). Single-crystal ENDOR study of a $57 \mathrm{Fe}$-enriched iron-sulfur $[4 \mathrm{Fe}-4 \mathrm{~S}]^{3+}$ cluster. J. Am. Chem. Soc. 111, 2464-2469.

Rossi, M., Pollock, W.B., Reij, M.W., Keon, R.G., Fu, R., and Voordouw, G. (1993). The hmc operon of Desulfovibrio vulgaris subsp. vulgaris Hildenborough encodes a potential transmembrane redox protein complex. J. Bacteriol. 175, 4699-4711.

Schürmann, P., Stritt-Etter, A.L., and Li, J. (1995). Reduction of ferredoxin:thioredoxin reductase by artificial electron donors. Photosynth. Res. 309, 309-312.

Setzke, E., Hedderich, R., Heiden, S., and Thauer, R.K. (1994). $\mathrm{H}_{2}$ :heterodisulfide oxidoreductase complex from Methanobacterium thermoautotrophicum: composition and properties. Eur. J. Biochem. 220, 139-148.
Shokes, J.E., Duin, E.C., Bauer, C., Jaun, B., Hedderich, R., Koch, J., and Scott, R.A. (2005). Direct interaction of coenzyme $\mathrm{M}$ with the active-site $\mathrm{Fe}-\mathrm{S}$ cluster of heterodisulfide reductase. FEBS Lett. 579, 1741-1744.

Simianu, M., Murakami, E., Brewer, J.M., and Ragsdale, S.W. (1998). Purification and properties of the heme- and iron-sulfur-containing heterodisulfide reductase from Methanosarcina thermophila. Biochemistry 37, 10027-10039.

Staples, C.R., Ameyibor, E., Fu, W., Gardet-Salvi, L., Stritt-Etter, A.L., Schürmann, P., Knaff, D.B., and Johnson, M.K. (1996). The function and properties of the iron-sulfur center in spinach ferredoxin: thioredoxin reductase: a new biological role for iron-sulfur clusters. Biochemistry 35, 11425-11434.

Staples, C.R., Gaymard, E., Stritt-Etter, A.L., Telser, J., Hoffman, B.M., Schürmann, P., Knaff, D.B., and Johnson, M.K. (1998). Role of the $\left[\mathrm{Fe}_{4}-\mathrm{S}_{4}\right]$ cluster in mediating disulfide reduction in spinach ferredoxin:thioredoxin reductase. Biochemistry 37 , 4612-4620.

Stojanowic, A., Mander, G.J., Duin, E.C., and Hedderich, R. (2003). Physiological role of the $F_{420}$-non-reducing hydrogenase (Mvh) from Methanothermobacter marburgensis. Arch. Microbiol. 180, 194-203.

Thauer, R.K. (1998). Biochemistry of methanogenesis: a tribute to Marjory Stephenson. Microbiology 144, 2377-2406.

Tietze, M., Beuchle, A., Lamla, I., Orth, N., Dehler, M., Greiner, G., and Beifuss, U. (2003). Redox potentials of methanophenazine and CoB-S-S-CoM, factors involved in electron transport in methanogenic archaea. ChemBioChem 4, 333-335.

Walters, E.M. and Johnson, M.K. (2004). Ferredoxin:thioredoxin reductase: disulfide reduction catalyzed via novel site-specific [4Fe-4S] cluster chemistry. Photosynth. Res. 79, 249-264.

Williams, C.H. (1995). Flavoprotein structure and mechanism. 6. Mechanism and structure of thioredoxin reductase from Escherichia coli. FASEB J. 9, 1267-1276.

Williams, C.H., Arscott, L.D., Muller, S., Lennon, B.W., Ludwig, M.L., Wang, P.F., Veine, D.M., Becker, K., and Schirmer, R.H. (2000). Thioredoxin reductase two modes of catalysis have evolved. Eur. J. Biochem. 267, 6110-6117. 